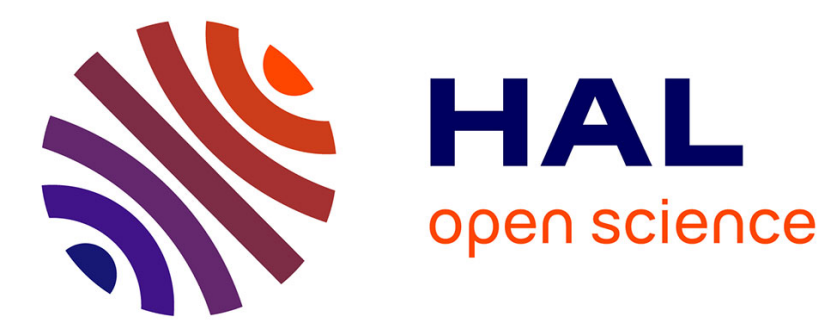

\title{
Aperçu de la dynamique de constitution de l'espace habité d'un village du Nord Viêt Nam
}

Olivier Tessier

\section{To cite this version:}

Olivier Tessier. Aperçu de la dynamique de constitution de l'espace habité d'un village du Nord Viêt Nam. Bulletin de l'Ecole française d'Extrême-Orient, 1996, 83 (1), pp.286 - 298. 10.3406/befeo.1996.3790 . halshs-02558342

\section{HAL Id: halshs-02558342 \\ https://shs.hal.science/halshs-02558342}

Submitted on 29 Apr 2020

HAL is a multi-disciplinary open access archive for the deposit and dissemination of scientific research documents, whether they are published or not. The documents may come from teaching and research institutions in France or abroad, or from public or private research centers.
L'archive ouverte pluridisciplinaire HAL, est destinée au dépôt et à la diffusion de documents scientifiques de niveau recherche, publiés ou non, émanant des établissements d'enseignement et de recherche français ou étrangers, des laboratoires publics ou privés. 


\section{Aperçu de la dynamique de constitution de l'espace habité d'un} village du Nord Viêt Nam

\section{Olivier Tessier}

\section{Citer ce document / Cite this document :}

Tessier Olivier. Aperçu de la dynamique de constitution de l'espace habité d'un village du Nord Viêt Nam . In: Bulletin de l'Ecole française d'Extrême-Orient. Tome 83, 1996. pp. 286-298;

doi : https://doi.org/10.3406/befeo.1996.3790

https://www.persee.fr/doc/befeo_0336-1519_1996_num_83_1_3790

Fichier pdf généré le 08/02/2019 


\section{VIÊT NAM}

\section{Aperçu de la dynamique de constitution de l'espace habité d'un village du Nord Viêt Nam}

Les résultats que nous présentons ici sont les premiers éléments d'un travail de recherche actuellement en cours, dont l'objectif est de proposer, à terme, une analyse historique et sociale des mécanismes de constitution de l'espace villageois dans quelques communes de la moyenne région du bassin du fleuve Rouge (Trung du) également appelée « région des collines » (Vùng đồi). En se fondant principalement sur les informations collectées auprès des habitants du village (thôn) de Hay, nous nous limiterons pour cette présentation à la description des principales étapes de la construction de l'espace habité de ce village ${ }^{1}$ qui compose avec ceux de Đồng Xa, Mánh et Xen, la commune de Ninh Dân (district de Thanh Ba, province de Vĩnh Phú).

L'organisation de l'espace villageois dans cette région se démarque nettement de celle des villages du delta du fleuve Rouge. Les nombreux auteurs qui se sont intéressés à cette plaine deltaïque décrivent le village « traditionnel » comme un îlot de verdure entouré d'une haie de bambous, véritable frontière séparant l'espace habité de l'espace agricole mais également limite de la communauté villageoise et symbole de son identité. Dans la région des collines, en revanche, l'espace habité et l'espace agricole se confondent, mélange qui donne à l'observateur extérieur l'impression d'un espace non structuré aux contours flous et évolutifs. P. Gourou décrit ainsi ces villages de bordure de collines « Dans ces villages se réalise un paysage exceptionnel : les maisons, largement séparées les unes des autres, sont établies sur des gradins taillés dans la colline, et devant chaque cour s'ouvre un large horizon. Une profusion de jacquiers, de lilas du Japon, de théiers couvre le sol et ombrage les sentiers abrupts qui gravissent les collines ${ }^{2}$. Cette description souligne l'importance de la parcelle d'habitation ou «jardin » que l'on peut définir comme l'espace de résidence constitué de la maison, des dépendances (cuisine, étables) et du jardin proprement dit (arbres fruitiers et à bois, thé, manioc, mare etc.), espace dont la superficie varie, pour ce village, de trois sào ${ }^{3}$ $\left(1000 \mathrm{~m}^{2}\right)$ à un mẫu $\left(3600 \mathrm{~m}^{2}\right)$, exceptionnellement plus.

1. Dans une publication ultérieure, nous proposerons une lecture critique de l'histoire de ce village telle qu'elle est retracée pas ses habitants, en se fondant pour cela sur les informations fournies par les archives communales (Archives nationales $n^{\circ} 1$, fonds de la Résidence de Phó Thọ) et sur la reconstitution de l'histoire d'autres villages du district de Thanh $\mathrm{Ba}$.

2. P. Gourou. Les paysans du delta tonkinois (Paris, E.F.E.O, 1936, 666 p.) p. 241-242.

3. Les unités de surfaces sont les suivantes : 1 thước $=24 \mathrm{~m}^{2} ; 1$ sào $0^{3}=360 \mathrm{~m}^{2}=15$ thước ; 1 mẫu $=3600 \mathrm{~m}^{2}=10$ sào. 

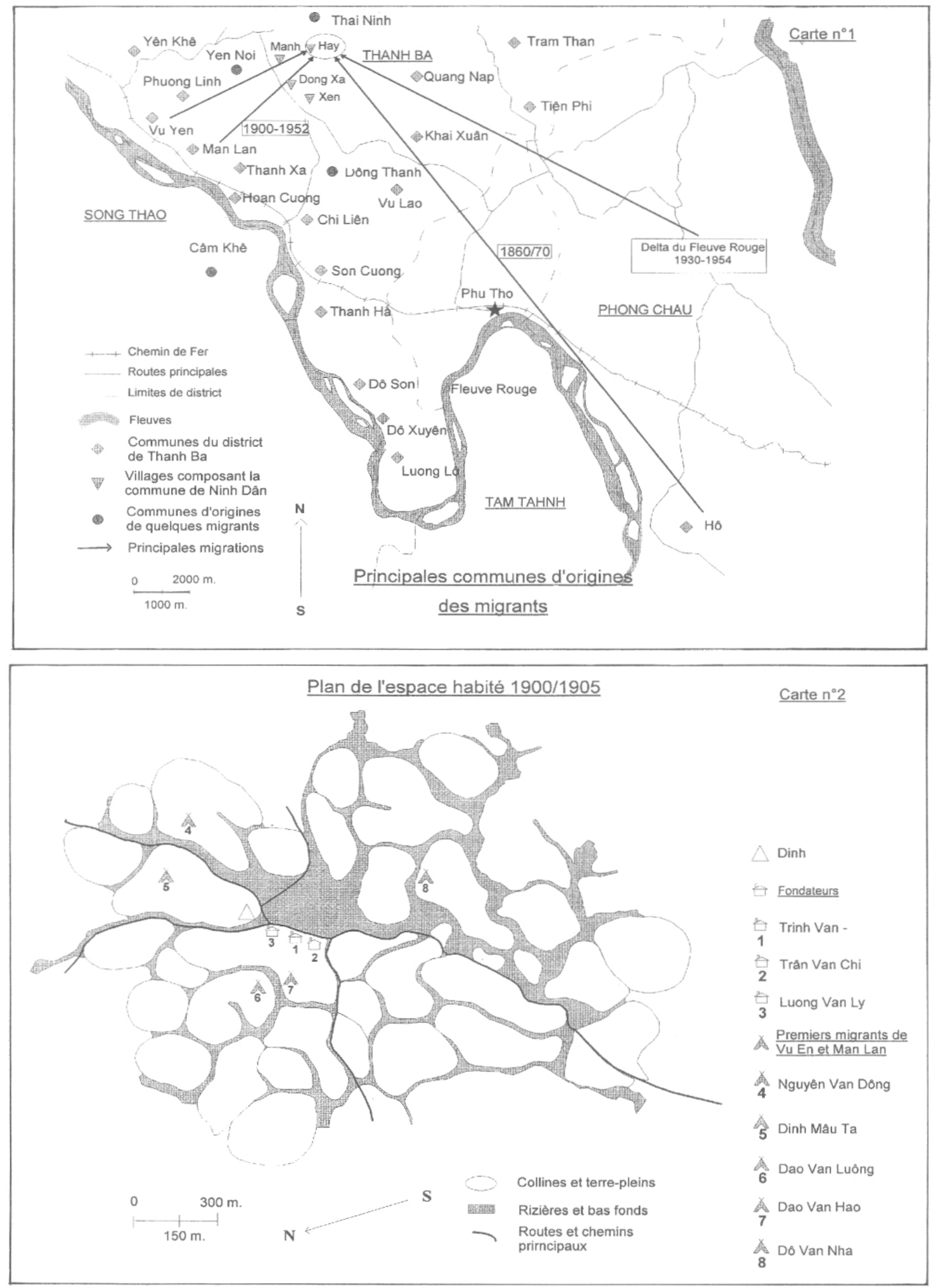

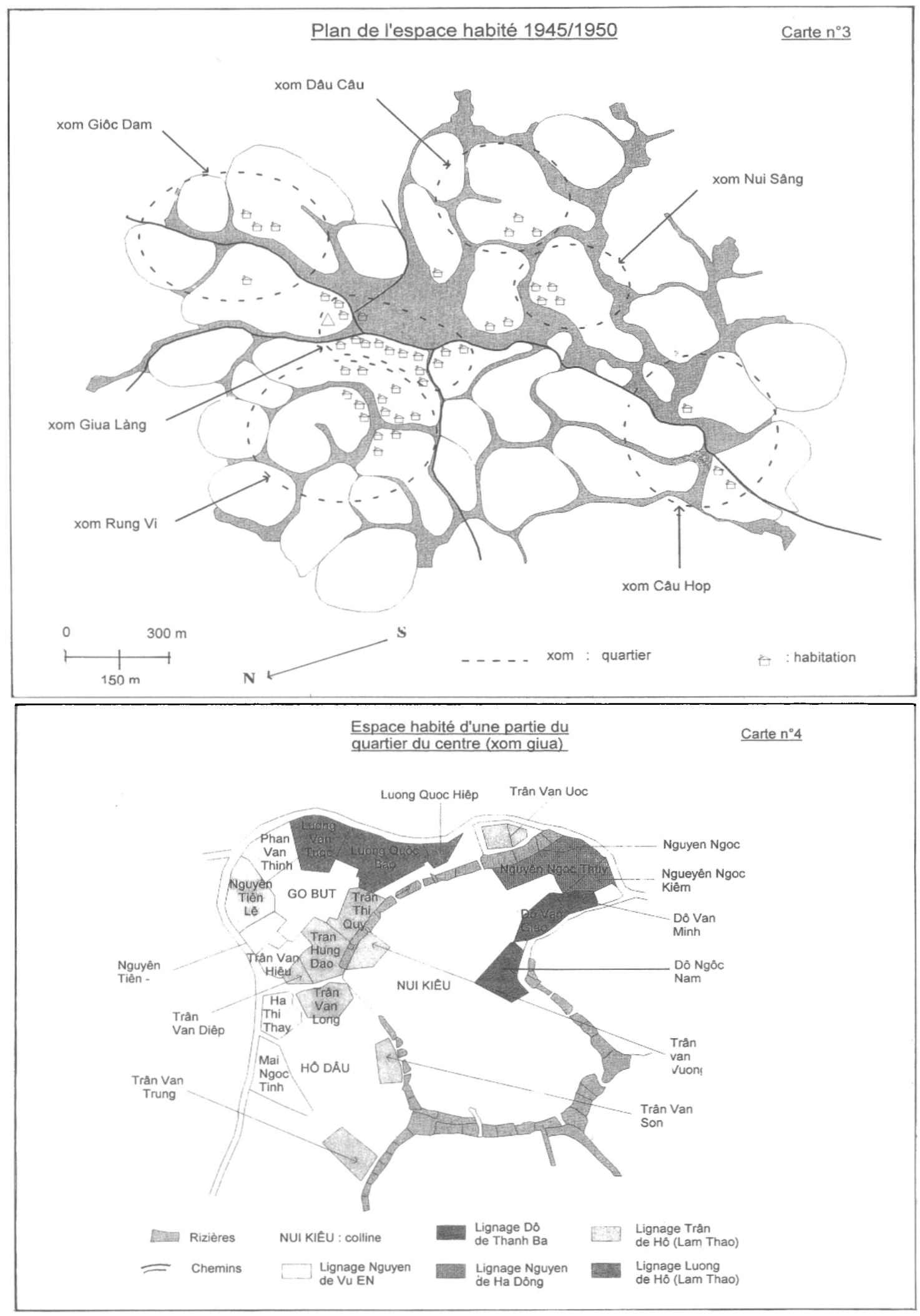
Lorsque nous évoquons l'histoire de ce village avec ses habitants, les conversations débutent fréquemment par cette phrase : « les gens de Hay viennent des quatre coins du monde » (đến từ tứ xứ). Elle illustre le fait que sa population actuelle est composée de migrants, et de leurs descendants, originaires d'autres villages du district de Thanh $\mathrm{Ba}$ ou d'autres provinces du delta du Fleuve Rouge. Ces migrations, qui sont à l'origine même de la création du village, se sont succédé jusqu'au début des années 1950 . À partir de l'histoire de ces migrations, nous essaierons de montrer que la structure actuelle de l'espace habité du village de Hay est le résultat, dans le cadre géographique spécifique de cette région, de l'interaction de divers facteurs d'ordre économique, politique, et social, facteurs dont l'influence respective fut plus ou moins prédominante suivant la période considérée sans pour autant qu'il soit possible d'en isoler un qui soit à même de « donner du sens à l'ensemble ».

\section{Première période : des origines à 1940}

\section{La création du village}

Les habitants actuels estiment que le village de Hay fut fondé au milieu du XIX ${ }^{\mathrm{e}}$ siècle par trois ressortissants (Trần Văn Chí, Lương Văn Lý et Trịnh Văn ${ }^{1}$ ) du village de Hồ (Phủ de Lâm Thao, actuel district de Phong Châu). Le fait que le village de Hay n'apparaisse pas dans la liste exhaustive des villages du nord Viêt Nam répertoriés entre 1810 et $1813^{2}$ confirme que sa reconnaissance officielle est postérieure au début du $\mathrm{XIX}^{\mathrm{e}}$ siècle. D'après les témoignages des descendants des trois fondateurs, ces derniers auraient pris la fuite lors d'une attaque de leur village (carte $n^{\circ} 1$ ) par les Cờ Đen (Pavillons Noirs) et se seraient réfugiés dans une région inhabitée qui est devenue, par la suite, le village de Hay. Le choix de cette zone enclavée (située à quatre kilomètres de la route) et éloignée du village de Lãng Hơ $(25 \mathrm{~km}$ à vol d'oiseau), l'inhospitalité du milieu en une période où l'exploitation des collines ne présentait pas encore d'intérêt particulier, sont autant d'éléments qui rendent plausible la version de la fuite, qui cadre par ailleurs avec l'histoire événementielle. En effet, les Pavillons Noirs furent, avec les Pavillons Rouges et les Pavillons Jaunes, des « pirates » chinois qui, suite à l'échec de la révolte des Taiping contre la domination mandchoue, se retirèrent au Tonkin, au milieu du XIX ${ }^{\mathrm{e}}$ siècle. L'historien Lê Thành Khôi évoque leur présence dans la province de Thái Nguyên dès 1851, année où ils furent réprimés par l'armée de l'Empereur Tù $Đ$ úc $^{3}$. Se pose maintenant la question de l'année approximative de l'arrivée des trois fondateurs, aucun écrit, dont nous ayons connaissance, ne mentionnant d'attaque de ces "pirates » dans le village de Lãng Hồ. Se fonder sur la seule période d'installation des Pavillons Noirs dans le delta du Fleuve Rouge, qui furent les derniers à occuper cette région après avoir repoussé les Pavillons Jaunes vers le Sud du Tonkin, semble hasardeux dans la mesure où les descendants des fondateurs ne font pas de distinction entre ces différentes bandes, Cờ Đen (Pavillons Noirs) étant pour eux un terme générique désignant l'ensemble des « pirates » chinois. Deux éléments nous permettent cependant de penser que leur arrivée s'est produite entre 1860 et 1880 . Le premier est lié au fait que durant cette période, les Pavillons Jaunes intensifièrent leur action de brigandage dans le delta. « Leur irruption sur le Fleuve Rouge en 1864 est une catastrophe qui entraîne, outre les pertes en vie humaine, l'exode des populations affolées, la ruine de l'économie et la misère pour de nombreuses années, encore

1. Le signet " - " indique que nous n'avons pu déterminer le nom complet de la personne.

2. Các trấn tổng xã danh bị. Texte repris dans : Tên Làng Xã Việt Nam đâu thế kỷ XIX', traduit par Dương Thị The et Phạm Thị Thoa (Noms des villages du Viêt Nam au début du XIXe siècle depuis le Nghệ Tỉnh jusqu'au Nord), Hà Nôi, Nhà xuất bản khoa học xã hội, 1981. p. 383.

3. Lê Thành Khôi, Histoire du Viêt Nam des origines à 1958 (Paris, Sudestasie, 1992, 452 p.) 
accrue si les bandes armées s'installent à demeure en vivant sur le pays qu'elles mettent en coupe réglée » ${ }^{1}$, et que les Pavillons Noirs, qui occupent tout le Nord Tonkin à partir de 1871, transforment la ville de Sơn Tây en forteresse ${ }^{2}$, ville qui se situe à seulement $30 \mathrm{~km}$ au sud du village de Lãng Hờ. Le second élément est fourni par la reconstitution des généalogies lignagères des fondateurs, dont l'année de naissance estimée se situe, pour deux d'entre eux, aux alentours de 1840, ce qui rend improbable une colonisation de la zone du futur village de Hay antérieurement à 1860 . Subsiste encore un doute sur la simultanéité de l'arrivée de Trần Văn Chí, Trịnh Văn et Lương Văn Lý, dont les descendants estiment qu'ils sont arrivés ensemble alors que d'autres habitants du village affirment que Lương Văn Lý n'est arrivé que quelques années plus tard. Leur communauté d'origine ainsi que les éléments étayant l'hypothèse de la fuite, nous incitent à penser que ces trois hommes ont quitté ensemble leur village d'origine.

Le besoin vital de création de rizières poussa ces trois hommes à coloniser une zone relativement plane, vaste, aisée à défricher et à mettre en valeur, ensemble de caractéristiques que seule la plaine actuelle du centre du village pouvait satisfaire (carte $n^{\circ} 2$ ). Ils y construisirent leurs habitations, toutes trois regroupées sur un terre-plein qui fait face à cette plaine. L'histoire de la colonisation du terroir du futur village de Hay débutait.

\section{La colonisation des zones périphériques}

Aux alentours des années 1920, est apparu un mode de colonisation original qui, s'il ne concernait au départ que quelques membres de quatre lignages distincts originaires des villages de Vũ En ${ }^{3}$ et de Mạn Lạn (carte ${ }^{\circ} 1$ ), a perduré jusqu'en 1952 et a participé à fixer les limites actuelles de l'espace villageois, ainsi qu'à déterminer son organisation spatiale interne. «Original », car ces colons « créèrent des campements » (làm trại) ${ }^{4}$ dans les collines périphériques du centre actuel du village de Hay, unique quartier habité en ce début de siècle, tout en conservant un lieu de résidence et des activités dans leur village d'origine.

La colonisation des zones périphériques, alors même que le centre du village n'était que peu densément peuplé, s'explique par les activités que souhaitaient développer ces colons : production de thé (cây chè) et, dans une moindre mesure, de laque (cây sơn) et de feuilles de latanier (cây lá nón). En effet, les terroirs respectifs des villages de Vũ En et de Mạn Lạn bordent le Fleuve Rouge et présentent des conditions agro-écologiques plus proches de celles de zones deltaïques (très faible densité de collines, vastes plaines alluvionnaires) que de celles de la moyenne région. La logique de mise en valeur du milieu de cette deuxième vague de colons était donc bien différente de celle des fondateurs. Dans la mesure où ils possédaient et exploitaient des rizières à Vũ En et Mạn Lạn, leur centre d'intérêt les conduisit à coloniser des zones à forte densité de collines, même si elles étaient pauvres en rizières potentielles (basfonds étroits et profonds). Ce mode de colonisation fut rendu possible par la faible distance séparant ces deux villages de celui de Hay (Vũ En $7 \mathrm{~km}$ et Mạn Lạn $8 \mathrm{~km}$, proximité qui permettait aux colons d'effectuer de fréquents aller-retours : Đinh Tiến Nghệ (62 ans) se

1. Gilles Raffi, Fleuve Rouge : de l'intérêt économique aux ambitions politiques (1857-1873) in Pays d'Outre-mer (Aix en Provence, Études et Documents n² 24, 1993, p. 109-133) p. 113.

2. Capitaine de Vaisseau Jouan, Courbet (1825-1885) (Hà Nội, Edition de l'Instruction publique en Indochine, 1942, 80 p.) p. 9.

3. Vũ En ou Vũ Yến sont deux orthographes admises.

4. L'expression làm trại, littéralement "créer des campements ", fait référence aux colons des fronts pionniers qui défrichèrent et colonisèrent progressivement le pays depuis le $\mathrm{X}^{\mathrm{e}}$ siècle, pour atteindre le delta du Mékong au XVII ${ }^{c}$. Cette expression illustre bien le mode de colonisation développé par les ressortissants de Vũ En et de Mạn Lạn qui ne résidaient que temporairement dans le village de Hay et dont les habitations étaient, tout au moins dans les premiers temps, frustres et sans confort. 
souvient que lorsqu'il était adolescent et élève à Vũ En, il lui fallait marcher environ deux heures pour rejoindre le village de Hay afin de rendre visite à ses parents.

Ces colons furent, d'une part, Đinh Mậu Tạ ${ }^{1}$ et Nguyễn Văn Đông tous deux originaires de Vũ En qui commencèrent, en 1902, à défricher les collines de l'actuel quartier de Dộc Giăm et, d'autre part, six membres du lignage Đào (Luông et cinq fils de ses deux frères) originaires du village de Mạn Lạn ainsi que Đỗ Văn Nhã originaire du village de Thanh Ba (intégré depuis à la commune de Mạn Lạn) qui firent de même, entre 1915 et 1920 respectivement dans les quartiers actuels de Rùng Vi et de Núi Sâng (carte $n^{\circ} 2$ ).

Pour comprendre cette deuxième étape de la constitution du village de Hay et les migrations qui s'y succédèrent jusqu'en 1950, comme dans d'autres villages du district de Thanh $\mathrm{Ba}$, il est nécessaire de les replacer dans le contexte général de l'époque. Dès la fin du $\mathrm{XIX}^{\mathrm{e}}$ siècle, la saturation progressive du foncier agricole disponible dans le delta du Fleuve Rouge, ainsi que dans les plaines alluvionnaires bordant les principaux cours d'eau, saturation qui s'est intensifiée au cours du $\mathrm{XX}^{\mathrm{e}}$ siècle, eut pour première conséquence d'hypothéquer la capacité d'autosuffisance alimentaire des couches de population les plus modestes de ces régions. « Pour des récoltes normales, la quantité de riz produite annuellement par les provinces du Bắc Viêt [ Nord Viêt Nam] suffit à peine aux besoins de la population. Il s'ensuit un état d'équilibre précaire, très vite rompu lorsque, pour une raison quelconque, les conditions du ravitaillement viennent à être modifiées " ${ }^{2}$. Cette situation préoccupante conduisit les autorités coloniales à définir, dès 1888 , une politique de colonisation de la moyenne et de la haute région. Si ces tentatives de colonisations directives (collectives ou individuelles) se soldèrent généralement par des échecs, leur existence même permet de prendre la mesure de la situation et de voir dans la colonisation du terroir du village de Hay une nécessité de diversification des productions et des sources de revenus. De plus, les facilités offertes par les autorités aux colons (usufruit permanent puis propriété de la terre défrichée, dispense d'impôts fonciers les premières années) ont sans nul doute favorisé les migrations spontanées et individuelles. Mais cette colonisation des zones de collines n'aurait sûrement pas eu la même ampleur sans la conjonction d'un second facteur : la croissance de la demande de certaines cultures de rente (principalement le thé). La moyenne région, dont les potentialités agricoles étaient jusqu'alors jugées peu attrayantes en regard des difficultés qu'engendrait sa mise en valeur, présentait des conditions agro-écologiques particulièrement bien adaptées à ce type de productions ${ }^{3}$ et suscita dès lors un vif intérêt. Le développement de ces cultures fut d'autant plus rapide ${ }^{4}$ qu'elles bénéficièrent de conditions favorables. La première fut la mise en place de mesures fiscales incitatives « Les autorités coloniales souhaitant développer l'exportation de thé noir vers la France, de thé vert vers la Chine et l'Afrique du Nord, et de laque vers le Japon, la taxe sur ces cultures est plus faible [que celle

1. Dans la suite de ce document, l'expression «membre du lignage Đinh » fera référence au lignage et au village d'origine du premier arrivant, en l'occurrence dans cet exemple, Đinh Mậu Tạ.

2. Nguyên Nhê Anh, La famine de 1945 au Nord Vietnam in Approche Asie (Paris, Puf, nº 28, p. 103-116) p. 103.

3. L'utilisation des produits forestiers (vente, autoconsommation) issus du défrichage est un point qui n'est pas évoqué par les descendants de ces colons bien que les premiers dépouillements des archives communales indiquent des transactions portant sur des billes de bois et des bambous.

4. Bien que D. Hemery et $P$. Brocheux, lorsqu'ils s'intéressent aux provinces productrices de thé destiné à l'exportation sous le protectorat français, ne mentionnent pas celle de Phó Thọ, l'existence à la veille de l'indépendance de deux unités de séchage de thé dans le village de Hay, employant une quinzaine d'ouvriers, ainsi que la création à la fin des années 1950 d'une usine d'État de séchage et de traitement du thé pour le seul district de Thanh Ba (dont dépend la commune de Ninh Dân), reflètent l'importance des surfaces plantées dans ce village et plus globalement dans ce district. D. Hemery et P. Brocheux, La colonisation ambiguë 1858-1954 (Paris, La découverte, 1995, 428 p.) p. 121. Ceci est illustré par les propos de Mme Nguyễn Thị Cụ (87 ans) : «Avant 1940 toutes les collines du village étaient couvertes de thé, il n'y avait rien d'autre ». 
touchant la riziculture ${ }^{1}{ }^{\prime}$. La seconde est liée à l'existence, à proximité des lieux de production, de voies de communication à même d'assurer le transport des marchandises vers Hà Nội. Il s'agit du Fleuve Rouge, qui n'est cependant pas navigable pendant la période de la mousson d'été, mais aussi du chemin de fer. La ligne ferroviaire du Yunan, qui fut construite entre 1901 et $1911^{2}$ et qui dans sa partie vietnamienne reliait Hà Nội à Lào Cai, desservait en effet les gares de Chí Chư et de Vũ Ẻn situées respectivement à cinq et sept kilomètres du village de Hay.

La forme originale de colonisation du milieu, commencée au tout début du $\mathrm{XX}^{\mathrm{e}}$ siècle par les habitants des villages de Mạn Lạn et de Vũ Ên se développa jusqu'à la fin des années 1940. Ce développement se fit, d'une part, par l'intermédiaire des descendants des premiers arrivants qui, en reproduisant le modèle initié par leur parents, défrichèrent et mirent en valeur de nouvelles terres de colline et, d'autre part, par l'arrivée de cinq nouveaux colons originaires de ces deux villages. Trois nouveaux lignages (Mè, Lê et Nguyễn) de Mạn Lạn eurent ainsi des membres établis dans le village. En revanche, seul Nguyễn Văn Nhân, membre du lignage Nguyễn de Vũ Ên précédemment établi, créa un campement aux alentours de 1930. Bien que l'intérêt économique que représentait la production de thé constituait la raison majeure motivant l'établissement de ces nouveaux campements, ceux-ci avaient également pour but de remédier à la déficience de rizières disponibles dans les villages de Vũ En et de Mạn Lạn, par la mise en valeur des vallées étroites et des terres de bas-fond. Ainsi, la colonisation du milieu n'était déjà plus, à cette époque, axée sur la seule exploitation des terres de collines, mais visait à développer un système de production associant cultures de rentes et riziculture.

Le développement rapide des surfaces cultivées en thé, qu'il ait été le fait des ressortissants de Vũ En et de Mạn Lạn ou des descendants des trois fondateurs, provoqua un appel de main-d'ocuvre extérieure, qui résultait de la conjonction des contraintes techniques qu'impose cette production et de la structure de la propriété foncière. En effet, l'exploitation du thé exige une main-d'œuvre importante ${ }^{3}$. La majeure partic des plantations étant détenue par un petit nombre de propriétaires (structure de la propriété foncière qui s'explique dans le cas de ce village par le mode de colonisation du milieu) l'ensemble des travaux ne pouvait être effectué par la seule main-d'œuvre familiale. Principalement originaires des provinces Thái Bình et Nam Hà situécs dans le delta du Fleuve Rouge, des salariés agricoles venaient seuls en laissant leurs familles dans leurs villages d'origine, logeaient directement dans la maison du propriétaire chez qui ils travaillaient, ne rentrant chez eux qu'une à deux fois l'an, pour le Tết notamment. Il s'agissait donc de migrations temporaires et non d'installations. Ils étaient rémunćrés à la tâche (làm khoán), se déplaçant de plantation en plantation au gré de la demande. En l'absence de données relatives à ces migrations temporaires, nous estimons qu'une trentaine de salariés était nécessaire à l'exploitation des 120 à 140 mâu (50 à 55 ha) de thé que comptait le village au début des années 1940, sachant qu'existaient, parallèlement aux plantations de plus de dix mẫu ( $3 \mathrm{ha}$ ), quelques petites exploitations ( 2 à 3 mẫu : 1 ha) que la main-d'ouvre familiale suffisait à mettre en valeur.

À la fin des années 1930, malgré l'installation des descendants des fondateurs et de trois nouveaux colons (1935/36), dont deux étaient originaires du district de Thanh Ba, la population de Hay était encore majoritairement «fluctuante », composée de salariés agricoles et de ressortissants de Vũ En et de Mạn Lạn qui conservaient un double lieu de résidence et une double activité.

1. M. Bousquet, Projet de recherche sur les systèmes agraires du bassin du Fleuve Rouge (Hà Nôi, GRET/ Programme Fleuve Rouge, 1992, 54 p.) p. 19.

2. D. Hemery et P. Brocheux, La colonisation ambiguë.... op. cité, p. 123.

3. J. L. Sabatier estime qu'une plantation d'un hectare mobilise 255 journées de travail par an pour être mise en valeur correctement. J. L. Sabatier, Gestion de l'eau dans la zone des collines de la Province de Vhin Phu au nord Vietnam (District de Than Hoa) (Communication non publiée/Tananarive/décembre 1991,16 p.) p. 8. 


\section{Une phase de profonds bouleversements}

À partir de 1940, les flux de population en direction de ce village et plus globalement du district de Thanh $\mathrm{Ba}$, n'eurent plus pour origine principale l'attrait économique que cette zone représentait depuis le début du siècle, mais furent également liés aux événements régionaux et nationaux qui se succédèrent jusqu'en 1954.

\section{De 1940 à 1952, l'arrivée d'un nombre croissant de migrants}

La situation de précarité, et ponctuellement de disette, dans laquelle se trouvait la paysannerie du delta du Fleuve Rouge, liée notamment à l'accroissement démographique et à la pénurie de terre qui en découlait, aggravée par de nombreuses inondations ${ }^{1}$ qui affectaient des provinces entières du delta, se dégrada rapidement sous l'occupation japonaise. « Les Japonais laissent en place l'administration coloniale ne faisant qu'une occupation stratégique et économique. Pour les Vietnamiens, c'est un double joug. Le riz est drainé vers le Japon. La misère s'aggrave d'autant plus que la colonie européenne, à partir de 1940, vivra sur les ressources du pays ${ }^{2}$. Son point culminant fut atteint au cours du printemps de l'année 1945, où une terrible famine causa la mort de plusieurs centaines de milliers de personnes ${ }^{3}$. Ces événements, auxquels vont succéder ceux de la première phase de la guerre d'Indochine (194750) furent à l'origine d'une vague de migration en direction de la moyenne région, qui affecta logiquement le village de Hay. Enfin, il est également nécessaire de tenir compte du fait que certaines arrivées purent être motivées par la volonté de ralliement à l'armée du Viêt minh, le village de Hay se trouvant dans la zone occupée par ses troupes, qui furent actives dès 19461947. Nous nous garderons donc de tout déterminisme exclusif, préférant parler d'un faisceau de facteurs dont chacun d'entre eux put être, suivant les cas, prépondérant dans la prise de décision de migrer.

Dans le cadre de cette vague de migration, nous avons répertorié neuf cas d'installations de salariés agricoles dans le village, installations que ces salariés ou leurs descendants, expliquent par leur mariage avec un(e) ressortissant(e) du village de Hay ou avec un(e) autre salarié(e). Mais si l'on observe leur date respective d'arrivée dans le village, force est de constater que deux seulement sont antérieures à 1940, les autres s'échelonnant de 1943 à 1949. Trois autres familles originaires du delta du Fleuve Rouge s'établirent également dans le village entre 1945 et 1947 et y développèrent des activités non agricoles : création d'une petite unité de séchage de thé (1947), et d'un atelier de tressage de paniers.

La succession d'événements décrits précédemment eut également des répercussions importantes sur le mode de colonisation et d'exploltation du milleu développé par les ressortissants des villages de Mạn Lạn et de Vũ En. Trois membres du lignage Đõ et cinq membres du lignage Đào exploitant une ferme à Hay, abandonnèrent respectivement les villages de Thanh Ba (commune de Mạn Lạn) en 1940 et de Mạn Lạn en 1950 pour se fixer à Hay. À la fin de cette année-là, les seuls ressortissants de cette commune qui pratiquaient encore une double activité en deux lieux distincts sont Mè Văn Cũ et ses enfants, pratique

1. Pour la scule période de $1890-1940$, D. Vesin a répertorié dix inondations majeures qui affectèrent des provinces entières du delta du Fleuve Rouge (années 1893,1904, 1905, 1909, 1911, 1913, 1915, 1926, 1937 et 1940). D. Vesin, Histoire du Fleuve Rouge. Gestion et aménagement du système hydraulique des années 1890 jusqu'à la seconde guerre mondiale (Paris, mémoire de maîtrise d'histoire, Université Paris VII, 1992, 329 p.) p. 39-47.

2. G. Challiand, Les paysans du nord Viêt-nam et la guerre (Paris, Cahier Libres $\mathbf{n}^{\circ} 130-131$ François Maspero, 1968, 195 p.) p. 28.

3. Le nombre de victimes est toujours actuellement sujet à controverse, variant d'un à deux millions suivant les auteurs. Voire notamment à ce sujet l'article de Nguyên Yhê Anh, La famine de 1945 ... op. cit., et l'ouvrage des professeurs Furata Moto et Văn Tạo Nạn Dói Nam 1945 ở Việt Nam (Hà Nội Xuất Bản Viẹn Sử Học Việt Nam, 1995, 727 p.). 
qu'ils abandonnèrent deux ans plus tard. En 1951-1952, les bombardements de l'aviation française sur la gare de Vũ Én ${ }^{1}$, qui ont, d'après les témoignages recueillis, affecté plusieurs quartiers du village, mirent un point final à cette pratique commencée quarante ans plus tôt. L'ensemble des membres des lignages Đinh et Nguyễn de Vũ Ến qui possédaient une ferme en zone de collines, quittèrent leur village natal pour s'installer définitivement à Hay. Ce mouvement ne se limita pas aux seuls villageois ayant auparavant une double activité. Entre 1948 et 1952, quatre ressortissants de Vũ Ẻn (dont trois du lignage Nguyễn), cinq de Mạn Lạn (lignage Trằn, Mè Lê, et Nguyễn) et trois d'autres communes du district de Thanh $\mathrm{Ba}$, qui n'avaient jusqu'alors développé aucune activité dans le village de Hay, s'y établirent.

À ce stade de la présentation, deux tendances relatives au mode de colonisation développé par les ressortissants de Vũ Én et Mạn Lạn et à ses conséquences sur la construction et l'organisation de l'espace habité du village de Hay peuvent être dégagées.

La première est que le mode de colonisation fondé sur la conservation d'activités et d'habitat dans le village d'origine ne concerna pas uniquement le village de Hay, des membres des lignages Mè et Trần de Mạn Lạn ayant également «créé des campements » dans la commune voisine de Thái Ninh. Mè Văn Hạai cite quatre communes du district de Thanh Ba (Đông Lĩnh, Đào Giã, Thái Ninh et Ninh Dân) dans lesquelles des membres de son lignage ont développé ce mode de colonisation, mouvement dont il explique ainsi l'origine : « Déjà à cette époque [1910-1930] il y avait trop de gens à Mạn Lạn et pas assez de terres pour les jeunes qui voulaient s'installer. Les paysans ont alors créé des campements dans d'autres régions comme à Ninh Dân par exemple ».

La seconde est que s'opéra un regroupement spatial des colons, en fonction de l'installation antérieure d'un membre du même lignage ou, si le nouvel arrivant était le premier de son lignage, d'une communauté d'origine avec des personnes précédemment installées. Ainsi, jusqu'à la fin des années 1940, le quartier Dộc Giăm était uniquement constitué de ressortissants de Vũ Ên, le quartier Rừng Vi d'habitants de Mạn Lạn et le quartier Núi Sâng de membres du lignage Đỗ de Thanh $\mathrm{Ba}\left(\right.$ carte $^{\circ} 3$ ). Le quartier du centre (X6́m Giứa) enfin, était encore principalement composé de membres des trois lignages fondateurs originaires du village de Hồ, bien qu'il comptait déjà quelques maisons de migrants récemment installés. Dans ce cadre, l'arrivée d'un nouveau colon et la détermination de son lieu d'installation en fonction de son appartenance lignagère (cas des lignages Lê, Nguyễn, Mè) et/ou de sa communauté d'origine avec un migrant précédemment installé (lignages Lê, Mè, Trần, Phan et Nguyễn) semble indiquer que ces deux unités (sociale et géographique), d'une part, furent utilisées comme des réseaux facilitant l'installation des nouveaux arrivants et, d'autre part, représentaient deux niveaux de reconnaissance identitaire suffisamment prégnants pour que se constituent des zones «d'exclusivité " ${ }^{2}$. En effet, entre 1940 et 1952, l'installation des migrants en provenance du delta du Fleuve Rouge ou de villages du district de Thanh $\mathrm{Ba}$ autres que ceux de Vũ Én et de Mạn Lạn, se fit généralement à l'extérieur des quartiers précédemment évoqués provoquant ainsi une extension de l'espace habité à des zones réservées jusqu'alors à la production agricole. Ce phénomène s'était déjà produit au milieu des années 1930, lors de l'arrivé de Vi Văn Thanh et Nguyễn Văn Dực qui furent les premiers à s'installer, respectivement, dans les quartiers actuels de Đầu Cầu et Cầu Hóp, alors que les autres quartiers n'étaient encore que peu densément peuplés.

1. Nous ne pouvons encore préciser ni la date exacte ni le(s) objectifs de ces bombardements, les personnes agées ainsi que les autorités de la commune interrogées à ce sujet proposant deux versions différentes. Pour les uns, cette opération visait à détruire la gare de Vũ Én afin de stopper le ravitailement en armes et en matériel en provenance de République populaire de Chine; pour les autres, c'était l'embarcadère situé sur le Fleuve Rouge qui en était la cible.

2 . Nous ne pouvons ici présenter les mécanismes ayant permis le maintien de ces «zones d'exclusivité », les investigations concernant ces pratiques n'étant pas encore achevées. 
En un peu plus de dix années, le nombre de foyers que comptait le village doubla quasiment, passant d'une trentaine en 1940, dont la majorité ne résidait que temporairement au village, à soixante-cinq en 1954, tous installés durablement. Le doublement de la population et l'abandon des terres agricoles de Vũ Én et de Mạn Lạn par les ressortissants de ces deux villages qui s'installaient progressivement à Hay, posa un problème de disponibilité en rizières dans un contexte de propriété foncière inégalement répartie. Le village fut libéré au cours de l'année 1952 et l'une des tâches auxquelles se consacrèrent les nouvelles autorités de la commune de Ninh Dân fut l'organisation de la réforme agraire qui se déroulera au milieu de l'année 1954.

\section{La réforme agraire}

La réforme agraire visait prioritairement une restructuration de la propriété foncière agricole, la propriété des parcelles d'habitation étant conservée en l'état, à l'exception de celle des «propriétaires terriens » (địa chử). Elle fut fondée sur la répartition des habitants en six classes, l'appartenance à chacune d'elles étant déterminée par la surface agricole possédée et la masse salariale employée, la classe de paysans moyens (trung nông) jouant le rôle de pivot autour duquel s'articulait la distribution ou au contraire la confiscation de terres, voire de biens. Nous nous bornerons ici à présenter les conséquences de cette réforme, sans entrer plus avant dans le détail du processus de classification ni des modalités de son application, d'autres critères liés au passé de chaque habitant ayant parfois été pris en compte, ce que s'est efforcé de corriger le décret de réhabilitation de 1956.

Pour le village de Hay, Trịnh Văn Hoè et Trần Văn Thư, descendants de deux fondateurs du village, furent déclarés « propriétaires terriens ». Leurs terres, leurs biens mobiliers et immobiliers furent confisqués, et ils furent tous deux déplacés dans le village voisin, Mánh. Leurs habitations furent distribuées à des «paysans pauvres » ( $b$ ần nông) ou à des salariés agricoles (paysans sans terre : cốnông) méritants (membre du comité de la réforme agraire, de l'Armée Populaire du Viêt Nam, etc.), généralement à raison de deux familles par maison'. Bien que la confiscation d'habitations ne concernât que ces deux grands propriétaires, cette réforme eut un impact non négligeable sur l'organisation de l'espace habité. D'une part, parce que des «paysans pauvres » et des «paysans sans terre " se virent attribuer une parcelle d'habitation, principalement dans le quartier du centre (Xóm Giữa), et, d'autre part, parce que Trằn Văn Thư possédait cinq maisons, dont trois dans ce quartier, et Trịnh Văn Hoè deux, soit un total de sept maisons qui furent toutes redistribuées. S'inscrivait ainsi dans l'espace, la volonté politique de rejet des valeurs de "l'ancienne vie », ce quartier n'abritant plus une majorité de descendants des fondateurs du village « propriétaires fonciers » mais, au contraire, une population récemment arrivée et jusqu'alors tenue à l'écart. Quantitativement, cette réforme eut également pour conséquence de concentrer dans le quartier du centre (Xóm Giưa) plus de la moitié de la population du village, prédominance qui s'atténuera cependant au fil du temps au profit des quartiers périphériques.

\section{Deuxième période : de 1960 à nos jours}

Depuis cette réforme, le village n'a pas connu, comme par le passé, de vagues de migrations significatives. L'évolution du contexte économique et les événements historiques d'ampleur nationale ne provoquèrent pas, comme par le passé, de remaniements conséquents de son espace habité, et cela malgré les profondes transformations et les événements tragiques qu'a connu le Nord Viêt Nam en cette deuxième moitié de $\mathrm{XX}^{\mathrm{e}}$ siècle. Au contraire, depuis

1. Cette situation se solda rapidement par la vente de la moitié de maison détenue par une des deux familles à l'autre famille. 
1960, le développement du village est avant tout dû à l'accroissement de sa propre population, la grande majorité des nouvelles installations enregistrées étant le fait de ressortissants de Hay qui se marient ${ }^{1}$, que le conflit avec les États-Unis eut pour conséquence de retarder. Les quelques cas d'installations exogènes sont généralement le fait de ressortissants de ce que l'on pourrait appeler la «zone de proximité du village», composée des autres villages de la commune de Ninh Dân et des communes limitrophes. Les mouvements de populations d'ampleur nationale, notamment les mouvements « tập kết » (migration de ressortissants de la République du Viêt Nam (Sud) vers la République Démocratique du Viêt Nam (Nord) d'août 1954 à mai-juin 1955) et «xây dùng quê hương mởi » (construire un nouveau pays, 19641965) ne drainèrent que peu de migrants vers Hay ${ }^{2}$. En définitive, l'installation à la fin des années 1980, de six anciens ouvriers qui travaillaient dans des entreprises et plantations d'État (également appelés les «176 $»^{3}$ ) constitue le seul mouvement de population conséquent que le village ait connu depuis la réforme agraire, mouvement qui s'apparente d'ailleurs à un « simple retour », ces anciens ouvriers étant tous originaires de Hay.

Ce faible nombre d'arrivées n'a pas pour autant figé la structure de l'espace habité. Cet espace s'est étoffé et a évolué en fonction de l'accroissement de la population, de la disponibilité du foncier et des options politiques adoptées par les différents gouvernements depuis 1960 , mais dans un cadre fixé par des pratiques d'installation qu'il est nécessaire de définir préalablement pour comprendre son évolution et son organisation actuelle. La caractéristique majeure est la tendance au regroupement spatial, sur une partie de colline, des habitations du père et de ses fils mariés et, dans quelques cas, de filles en situations particulières (divorce, cas d'uxorilocalité), regroupement à l'intérieur duquel les habitations restent indépendantes et possèdent chacune une cuisine. Si ce regroupement n'est pas systématique, il est le niveau d'organisation de l'espace habité le plus répandu dans le village.

Pendant la période de collectivisation des outils et des moyens de production (1959-1960 et 1981-1987) et jusqu'à la dernière redistribution des terres de 1992, le Comité Populaire de Ninh Dân attribuait aux couples ressortissants de la commune qui désiraient s'établir dans le village de Hay, un lopin de terre de colline (deux à trois sào : de 600 à $1000 \mathrm{~m}^{2}$ ) destiné à constituer leur parcelle d'habitation (maison et jardin). Dans la mesure où les autorités tenaient compte des vœux émis par le demandeur, qui souhaitait généralement obtenir une parcelle d'habitation proche de celle de ses parents, se sont constitués des regroupements dont la surface totale augmentait à chaque nouvelle installation. Au fil des attributions de parcelles d'habitation s'est inévitablement posé un problème de saturation de l'espace habitable, notamment dans le quartier du centre, les terres à vocation agricole ne pouvant être transformées en terres constructibles qu'au terme d'une procédure longue et incertaine. Dès la fin des années 1970, deux variantes aux pratiques d'installations définies précédemment virent le jour. Dans le cadre de la première, le fils marié s'installait sur une partie de la parcelle d'habitation du père, division qui n'entraînait plus d'augmentation de la surface du regroupement mais qui conservait et renforçait ce dernier. Au contraire, dans le cadre de la seconde, les jeunes couples s'installaient en périphérie des quartiers existants ou dans des quartiers encore peu densément peuplés afin de ne pas perdre l'avantage de l'attribution de la parcelle d'habitation mais cela, bien sûr, au détriment de la constitution du regroupement.

1. Nous parlons ici des hommes, les femmes pouvant être originaires d'autres villages, communes ou provinces.

2. Dans le cadre du premier mouvement, seul un militaire du sud s'est installé à Hay, région d'origine qu'il a d'ailleurs rejoint dès la réunification du pays en 1975. Pour le second, bien que les villageois évoquent une dizaine de tentatives d'installations, seules deux familles se sont établies durablement dans le village.

3. Les « 176 » sont les fonctionnaires et ouvriers des entreprises d’État qui furent mis à la retraite ou licenciés à partir de 1989, en application de la directive d'État $n^{\circ} 176$. 
Depuis la dernière redistribution des terres de $1992^{1}$ et la politique de monétarisation du foncier qui l'accompagna ${ }^{2}$, la pratique de division de la parcelle d'habitation du père, afin de permettre l'installation des fils nouvellement mariés, s'est progressivement généralisée et touche maintenant des zones où des terres constructibles sont encore disponibles. En effet, les parcelles d'habitation ne sont plus attribuées mais vendues par le Comité Populaire, et bien que les prix restent peu élevés par rapport à ceux pratiqués dans le reste de la commune ${ }^{3}$, les 300.000 à 500.000 đồng ( 150 à 250 francs) que coûte un sào de jardin représentent pour les jeunes couples mariés sans capital une somme importante dont ils ne disposent généralement pas. Il n'y a donc plus constitution d'un patrimoine foncier mais division de celui du père. La taille des parcelles d'habitation actuelles ne permet cependant pas d'envisager des divisions « sans fin », qui ramèneraient progressivement l'espace d'habitation à celui occupé par la maison et la cuisine. En effet, dans la moyenne région, cet espace joue un rôle important pour l'alimentation et l'économie des ménages. Cette tendance à la division n'est donc qu'une étape temporaire et ne pourra vraisemblablement pas se reproduire avec la même intensité pour la génération suivante, c'est-à-dire pour les enfants des jeunes couples qui se sont installés depuis quatre ans.

Pour conclure cette présentation, nous allons retracer brièvement l'histoire de la constitution d'une fraction du quartier du centre (collines de Gò Bụt, Núi Kiêu et Hố Dâu), description qui illustre les étapes majeures de la construction de l'espace habité du village de Hay (carte $\left.n^{\circ} 4\right)$.

Jusqu'au milieu des années 1940, seuls Lương Văn Lễ et Lương Văn Trà (terrain actuellement occupé par son fils Lương Văn Tước), tous deux petits fils du fondateur Lương Văn Ly, étaient établis sur la colline Gò Bụt qui appartenait à leur frère aîné Lương Văn Tiến. La colline Núi Kiêu ne comptait qu'une habitation, celle de Trần Văn Phấn, fils du «propriétaire terrien » Trần Văn Thư. À la fin des années 1940, Lê Kim Cương (originaire Mạn Lạn) et Nguyễn Văn Thị s'installèrent respectivement sur la colline Hố Dâu et Gò Bụt.

À partir de la réforme agraire, cette organisation relativement simple fut profondément modifiée. Mai Ngọc Ninh et Phan Văn Tường, respectivement salarié agricole et « paysan pauvre ", reçurent deux parcelles d'habitation occupées actuellement par leurs fils Phan Văn Thỉnh et Mai Ngọc Thịnh. Ces deux parcelles étaient celles de Lê Kim Cương, à qui l'on attribua une maison d'un des fils de Trần Văn Thư (Trần Văn Đạt), et de Lương Văn Lẽ décédé en 1953 sans avoir d'enfant. Nguyễn Văn Thị regagna en 1954 son village natal abandonnant sa parcelle d'habitation qui resta inoccupée jusqu'en 1965-1966. Deux autres maisons de Trần Văn Thư (terrains actuels de Đỗ Văn Giao et de Nguyễn Ngọc Thụy) furent distribuées à quatre « paysans pauvres » (deux familles par maison) qui les vendirent par la suite.

L'espace habité s'est ensuite étoffé au rythme des installations des couples nouvellement mariés à qui le Comité Populaire de la commune attribuait une parcelle d'habitation : Lương Quốc Bao en 1963, Trần Hưng Đạo et Nguyễn Tiến Lễ en 1965-1966, Trần Văn Hiểu en 1974 et Trần Văn Long en 1976. Après une série de cessions relativement complexes, les deux anciennes habitations du "propriétaire terrien » Trần Văn Thư furent rachetées par Nguyễn Ngọc Thụy en 1972 et Đỗ Văn Giao en 1976. Enfin, Trần Thị Quý, dont le mari ne réside pas

1. Schématiquement, chaque foyer paysan a l'usufruit des terres qui lui furent attribuées pour une durée de 10 à 20 ans en ce qui concerne les rizières et pour 50 ans en ce qui concerne les terres de collines.

2. Bien que la vente des parcelles d'habitation ne débuta officiellement qu'en 1992 pour la commune de Ninh Dân, elle fut effective dès 1991 (entretien avec le président du Comité Populaire de la commune).

3. Dans le village de Đông Xa (centre de la commune) en bordure de route, les parcelles loties par le Comité Populaire, sont vendues de 10 à 15 millions de đông les $100 \mathrm{~m}^{2}$. 
au village (chauffeur au siège du Comité Populaire de la province de Vĩnh Phú) s'installa en 1980-1981 sur un terrain contigu à celui de son frère Trân Hưng Đạo.

L'installation des descendants de la génération qui s'est établie entre 1960 et 1975 débuta, pour cette zone, en 1987. Deux fils de Trần Văn Học (frère aîné de Trân Hưng Đạo), Trân Văn Sơn et Trân Văn Trung, qui ne purent obtenir de parcelles d'habitation jouxtant celle de leur père, s'installèrent en 1987 et 1990 sur la colline Hó Dâu. En revanche, en 1988, Trăn Văn Vượng obtint du Comité Populaire de la commune un terrain faisant face à celui de ses parents (Trần Hưng Đạo). En l'absence de terrains constructibles à proximité de leurs parcelles, Lương Quớc Bao et Đỗ Văn Giao divisèrent leurs jardins afin de permettre l'installation de leurs fils, Lương Quó́c Hiệp et Đỗ Van Minh en 1989, et Đỗ Ngọc Nam en 1990. Depuis la redistribution du foncier de 1992, cinq nouvelles installations ont eu lieu. Dans quatre cas, le jeune couple s'est installé sur une partie du jardin du père du jeune marié [Trần Văn Điệp (1994), Nguyễn Ngọc Kiêm (1995), Nguyễn Ngọc Kiểm (1996) et Nguyễn Tiến Lễ (1996)] Seul Trần Văn Ước et sa femme, tous deux licenciés d'une ferme d'État en 1992, achetèrent un terrain en bordure de la route afin d'y ouvrir une épicerie, investissement que leurs primes de licenciement ont permis de financer. 